\title{
Solution of multiplicative homogeneous linear differential equations with constant exponentials
}

\author{
Numan Yalcin ${ }^{1}$ and Ercan Celik ${ }^{2}$ \\ ${ }^{1}$ Department of Mathematical Engineering, Gumushane University, Gumushane, Turkey, \\ ${ }^{2}$ Department of Mathematics, Ataturk University, Erzurum, Turkey
}

Received: 30 November 2017, Accepted: 20 March 2018

Published online: 29 March 2018.

\begin{abstract}
In this work, taking solutions of homogeneous differential equations with constant coefficients in classical analysis as a basis, solutions of homogeneous differential equations with constant exponentials in multiplicative analysis are obtained. Thus, solutions for these equations, being a class of non-linear differential equations, and having correspondence in the classical sense are stated.
\end{abstract}

Keywords: Multiplicatively linear independent functions, multiplicatively Wronskian, multiplicative homogeneous linear differential equations.

\section{Introduction}

Beginning from last century non-Newtonian calculus have developed and many researchers pay attention to this branch. In non-Newtonian calculus, differentiation and integration are based on non-Newtonian operations instead of classical operations. Grossman and Katz, introduced the non-Newtonian calculus consisting of the branches of geometric, anageometric and biogeometric calculus, etc. Fundamental definitions and concepts related to Non-Newtonian calculus are given in [1]. Geometric calculus is named as Multiplicative calculus after Dick Stanley [2]. Multiplicative calculus uses "multiplicative derivative" and "multiplicative integral". Thus it is an alternative to the classical calculus of Newton and Leibniz (also referred as Newtonian calculus), which has an additive derivative and an additive integral. In [3], Bashirov and friends gave further concepts and applications to the properties of derivative and integral operators of the multiplicative calculus. Some studies in recent years, [4-12] the multiplicative analysis, can be used in solving some of the problems in science and engineering, and illustrates the problems can be solved in a more practical way with the help of this analysis. In this study, the solution of multiplicative homogeneous differential equations with constant exponentials is researched. For this purpose, first of all, multiplicatively linearly independent, multiplicatively linearly dependent and Wronskian determinant of the functions are expressed. After that, the analysis, which is similar the solution of classical homogeneous linear differential equations with constant coefficients, are generalized to the multiplicative homogeneous differential equations with constant exponentials. Hence, solutions of a class of nonlinear differential equations which is multiplicative homogeneous linear differential equations with constant exponentials are obtained.

\section{Multiplicative Derivatives}

Here, we will give some basic definitions and properties of the multiplicative derivative theory which can be found in [2-4]. 
Definition 1. Let $f: R \rightarrow R^{+}$be a positive function. The multiplicative derivative of the function $f$ is given by:

$$
\frac{d^{*} f}{d t}(t)=f^{*}(t)=\lim _{h \rightarrow 0}\left(\frac{f(t+h)}{f(t)}\right)^{\frac{1}{h}} .
$$

Assuming that $f$ is a positive function and using properties of the classical derivative, multiplicative derivative can be written as

$$
\begin{aligned}
& \frac{d^{*} f}{d t}(t)=f^{*}(t)=\lim _{h \rightarrow 0}\left(\frac{f(t+h)}{f(t)}\right)^{\frac{1}{h}}, \\
& \frac{d^{*} f}{d t}(t)=f^{*}(t)=\lim _{h \rightarrow 0}\left(1+\frac{f(t+h)-f(t)}{f(t)}\right)^{\frac{f(t)}{f(t+h)-f(t)} \frac{f(t+h)-f(t)}{h} \frac{1}{f(t)}}, \\
& \frac{d^{*} f}{d t}(t)=f^{*}(t)=\lim _{h \rightarrow 0}\left(\left(1+\frac{f(t+h)-f(t)}{f(t)}\right)^{\left(\frac{f(t)}{f(t+h)-f(t)}\right)}\right)^{\left(\frac{f(t+h)-f(t)}{h} \frac{1}{f(t)}\right)}, \\
& \frac{d^{*} f}{d t}(t)=f^{*}(t)=e^{\frac{f^{\prime}(t)}{f(t)}}, \\
& \frac{d^{*} f}{d t}(t)=f^{*}(t)=e^{(\ln \circ f)^{\prime}(t)}
\end{aligned}
$$

where $(\ln \circ f)(t)=\ln (f(t))$.

Definition 2. If multiplicative derivative $f^{*}$ as a function has also multiplicative derivative then multiplicative derivative of $f^{*}$ is called second order multiplicative derivative of $f$ and it is represented by $f^{* *}$. Similarly we can define $n^{\text {th }}$ order multiplicative derivative of $f$ with the notation $f^{*(n)}$. With $n$ times repeated multiplicative differentiation operation, a positive $f$ function has an nth order multiplicative derivative at the point $t$ and defined as

$$
f^{*(n)}(t)=e^{(\ln \circ f)^{(n)}(t)}
$$

Theorem 1. If a positive function $f$ is differentiable with the multiplicative derivative at the point $t$, then it is differentiable in the classical sense and the relation between these two derivatives can be shown as

$$
f^{\prime}(t)=f(t) \ln f^{*}(t)
$$

Theorem 2. Let $f$ and $g$ be differentiable with the multiplicative derivative. If $c$ is an arbitrary constant, then $c . f, f . g$, $f+g, f / g, f^{g}$ functions are differentiable with the multiplicative derivative and their multiplicative derivatives can be shown as

(1) $(c . f)^{*}(t)=f^{*}(t)$,

(2) $(f \cdot g)^{*}(t)=f^{*}(t) \cdot g^{*}(t)$,

(3) $(f+g)^{*}(t)=f^{*}(t)^{\frac{f(t)}{f(t)+g(t)}} g^{*}(t)^{\frac{g(t)}{f(t)+g(t)}}$,

(4) $(f / g)^{*}=f^{*}(t) / g^{*}(t)$

(5) $\left(f^{g}\right)^{*}(t)=f^{*}(t)^{g(t)} f(t)^{g^{\prime}(t)}$.

Theorem 3. $f^{*}(t)=1$ for $\forall t \in(a, b) \Leftrightarrow f(t)=C>0$ is a fixed function in the open interval $(a, b)$.

Theorem 4. Let $g$ be differentiable in meaning of the multiplicative derivative and let $f$ be differentiable in the classical sense. If $f(t)=(g \circ h)(t)$, then, it can be written that

$$
f^{*}(t)=\left[g^{*}(h(t))\right]^{h^{\prime}(t)} .
$$


Theorem 5. Let $f$ be a positive function. Then, $f^{*}(t)=1 \Leftrightarrow f^{\prime}(t)=0$.

\section{Multiplicative linear differential equations}

Definition 3. Multiplicative linear differential equations can be defined in the form of

$$
\left(y^{*(n)}\right)^{a_{n}(t)}\left(y^{*(n-1)}\right)^{a_{n-1}(t)} \ldots\left(y^{* *}\right)^{a_{2}(t)}\left(y^{*}\right)^{a_{1}(t)} y^{a_{0}(t)}=f(t) .
$$

Here, $f(t)$ is a positive definite function. If, all of $a_{n}(t)$ exponentials are constants, equation (11) is called as multiplicative linear differential equations with constant exponentials. Otherwise, equation (11) is as called multiplicative linear differential equations with variable exponentials. In equation $(11)$, if $f(t)=1$, equation (11) is called multiplicative homogeneous linear differential equations, otherwise it is called multiplicative nonhomogeneous linear differential equations. Now, let's search how to solve these multiplicative homogeneous linear differential equations with constant exponentials. We describe multiplicatively linear independent and multiplicatively Wronskian determinant of positive definite functions.

Definition 4. Let $y_{1}, y_{2}, \ldots, y_{n}$ be positive definite functions. Any expression of the form

$$
y_{1}{ }^{c_{1}} y_{2}{ }^{c_{2}} \ldots y_{n}{ }^{c_{n}}
$$

is called a multiplicatively linear combinations of $y_{1}, y_{2}, \ldots, y_{n}$ where $c_{1}, c_{2}, \ldots, c_{n}$ are arbitrary constants[10].

Definition 5. Let $y_{1}, y_{2}, \ldots, y_{n}$ be positive definite functions. Then, they are called multiplicatively linear dependent if there are not all zero constants $c_{1}, c_{2}, \ldots, c_{n}$ with

$$
y_{1}(t)^{c_{1}} y_{2}(t)^{c_{2}} \ldots y_{n}(t)^{c_{n}}=1
$$

for all $t$. Otherwise, they are called multiplicatively linear independent.

Definition 6. Let $y_{1}, y_{2}, \ldots, y_{n}$ be at least $(n-1)$ times multiplicative differentiable positive definite functions. The determinant

$$
W_{m}\left(y_{1}, y_{2}, \ldots, y_{n}\right)=\left|\begin{array}{cccc}
\ln y_{1} & \ln y_{2} & \ldots & \ln y_{n} \\
\ln y_{1}{ }^{*} & \ln y_{2}^{*} & \ldots & \ln y_{n}{ }^{*} \\
\vdots & \vdots & \ddots & \vdots \\
\ln y_{1}^{*(n-1)} & \ln y_{2}^{*(n-1)} & \ldots & \ln y_{n}^{*(n-1)}
\end{array}\right|
$$

is called multiplicatively Wronskian determinant of the functions $\left\{y_{i}\right\}_{i=1}^{n}$.

Theorem 6. Let $y_{1}, y_{2}, \ldots, y_{n}$ be multiplicative differentiable positive definite functions on $[a, b]$. If multiplicatively Wronskian $W_{m}\left(y_{1}, y_{2}, \ldots, y_{n}\right)$ is nonzero for some $t_{0} \in[a, b]$, then these functions are multiplicatively linearly independent on $[a, b]$. If $y_{1}, y_{2}, \ldots, y_{n}$ are multiplicatively linearly dependent then the multiplicatively Wronskian is equal to zero for $\forall t \in[a, b]$.

Proof. Suppose that $y_{1}, y_{2}, \ldots, y_{n}$ are multiplicative differentiable positive definite functions on $[a, b]$. Let $W_{m}\left(y_{1}, y_{2}, \ldots, y_{n}\right) \neq 0$ for some $t_{0} \in[a, b]$, and assume the functions are multiplicatively linear dependent. Then, there exist arbitrary constants $c_{1}, c_{2}, \ldots, c_{n}$ not all equal to zero such that

$$
y_{1}{ }^{c_{1}} y_{2}{ }^{c_{2}} \ldots y_{n}{ }^{c_{n}}=1
$$


for $\forall t \in[a, b]$. Taking $(n-1)$ multiplicative derivatives of this equality gives us the following system of equalities

$$
\begin{aligned}
& y_{1}{ }^{c_{1}} y_{2}{ }^{c_{2}} \ldots y_{n}{ }^{c_{n}}=1 \text {, } \\
& y_{1}^{* c_{1}} y_{2}^{* c_{2}} \ldots y_{n}^{* c_{n}}=1 \text {, } \\
& y_{1}^{*(n-1)^{c_{1}}} y_{2}^{*(n-1)^{c_{2}}} \ldots y_{n}^{*(n-1)^{c_{n}}}=1 \text {. }
\end{aligned}
$$

Now, let's take the natural logarithm of both sides of equalities. So,

$$
\begin{gathered}
c_{1} \ln y_{1}+c_{2} \ln y_{2}+\ldots+c_{n} \ln y_{n}=0, \\
c_{1} \ln y_{1}^{*}+c_{2} \ln y_{2}^{*}+\ldots+c_{n} \ln y_{n}^{*}=0, \\
\vdots \\
c_{1} \ln y_{1}^{*(n-1)}+c_{2} \ln y_{2}^{*(n-1)}+\ldots+c_{n} \ln y_{n}^{*(n-1)}=0,
\end{gathered}
$$

Since, $W_{m}\left(y_{1}, y_{2}, \ldots, y_{n}\right) \neq 0$ for some $t_{0} \in[a, b]$. It follows that $c_{1}=c_{2}=\ldots=c_{n}=0$. But, this is in contradiction with $c_{1}, c_{2}, \ldots, c_{n}$ being not all equal to zero. So, functions are multiplicatively linearly independent.

Example 1. Consider $y_{1}=e^{e t}, y_{2}=e^{e^{-t}}, y_{3}=e^{e^{2 t}}$. Multiplicatively Wronskian determinant of these functions are

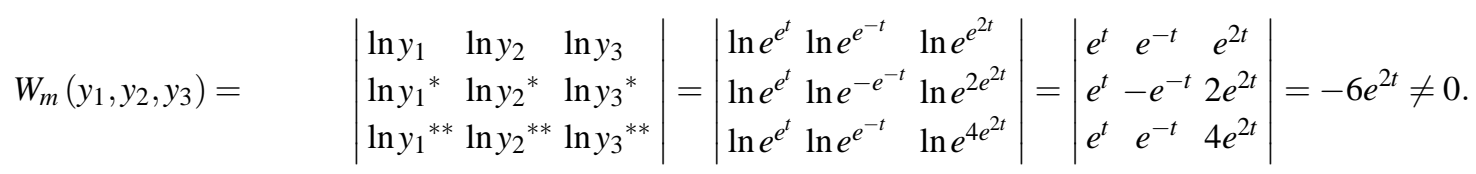

Thus, these functions are multiplicatively linear independent.

Example 2. For $t>0$, consider functions $y_{1}=t^{-1}, y_{2}=t, y_{3}=t^{2}$. Multiplicatively Wronskian determinant of these functions are

$$
W_{m}\left(y_{1}, y_{2}, y_{3}\right)=\left|\begin{array}{lll}
\ln \left(t^{-1}\right) & \ln t & \ln t^{2} \\
\ln \left(e^{-1 / t}\right) & \ln \left(e^{1 / t}\right) & \ln \left(e^{2 / t}\right) \\
\ln \left(e^{1 / t^{2}}\right) & \ln \left(e^{-1 / t^{2}}\right) & \ln \left(e^{-2 / t^{2}}\right)
\end{array}\right|=\left|\begin{array}{rrr}
-\ln t & \ln t & 2 \ln t \\
-\frac{1}{t} & \frac{1}{t} & \frac{2}{t} \\
\frac{1}{t^{2}} & -\frac{1}{t^{2}} & -\frac{2}{t^{2}}
\end{array}\right|=0 .
$$

Thus, these system of functions have multiplicatively linear dependent property.

\section{Multiplicative homogeneous linear differential equations with constant exponentials}

Now, we analyze solutions of multiplicative homogeneous linear differential equation with constant exponentials

$$
\left(y^{*(n)}\right)^{a_{n}}\left(y^{*(n-1)}\right)^{a_{n-1}} \ldots\left(y^{* *}\right)^{a_{2}}\left(y^{*}\right)^{a_{1}} y^{a_{0}}=1
$$

where $a_{k}, k=1, \ldots, n$ are the arbitrary constants. Class of this equation corresponds to the class of non-linear equations in the classical sense

$$
a_{n} \frac{d^{n-1}}{d t^{n-1}}\left(\frac{1}{y} \frac{d y}{d t}\right)+a_{n-1} \frac{d^{n-2}}{d t^{n-2}}\left(\frac{1}{y} \frac{d y}{d t}\right)+\ldots+a_{2} \frac{d}{d t}\left(\frac{1}{y} \frac{d y}{d t}\right)+a_{1}\left(\frac{1}{y} \frac{d y}{d t}\right)=-a_{0} \ln y .
$$


So, solutions of the equation (19) corresponds to solutions of the non-linear equation (20). By the help of following multiplicative derivative operators

$$
\begin{gathered}
y^{*}=\tilde{D} y=e^{D(l n y)}=e^{\frac{D(y)}{y}}, \\
y^{* *}=\tilde{D}^{2} y=e^{D^{2}(\operatorname{lny})}=e^{D\left(\frac{D(y)}{y}\right)}, \\
\vdots \\
y^{*(n)}=\tilde{D}^{n} y=e^{D^{n}(l n y)}=e^{D^{(n-1)}\left(\frac{D(y)}{y}\right)}, \\
L^{*}(\tilde{D})=\left(\tilde{D}^{n}\right)^{a_{n}}\left(\tilde{D}^{n-1}\right)^{a_{n-1}} \ldots(\tilde{D})^{a_{1}}\left(\tilde{D}^{0}\right)^{a_{0}}
\end{gathered}
$$

equation (19) can be rewritten as

$$
L^{*}(\tilde{D}) y=1
$$

Since $a_{0}, a_{1}, \ldots, a_{n}$ exponentials are constants, operator

$$
L^{*}(\tilde{D})=\left(\tilde{D}^{n}\right)^{a_{n}}\left(\tilde{D}^{n-1}\right)^{a_{n-1}} \ldots(\tilde{D})^{a_{1}}\left(\tilde{D}^{0}\right)^{a_{0}}
$$

also has constant coefficients.

Theorem 7. If equation (19) has multiplicatively linear independent solution $y_{1}(t), y_{2}(t), \ldots, y_{n}(t)$, then the general solution has the form

$$
y=y_{1}{ }^{c_{1}} y_{2}{ }^{c_{2}} \ldots y_{n}{ }^{c_{n}}
$$

where $c_{1}, c_{2}, \ldots, c_{n}$ are arbitrary constants.

Proof. Let $y_{1}(t), y_{2}(t), \ldots, y_{n}(t)$ be solutions of the equation (19). For arbitrary constants $c_{k}, k=1, \ldots, n$, introduce notation

$$
y=\left(y_{1}\right)^{c_{1}}\left(y_{2}\right)^{c_{2}} \ldots\left(y_{n}\right)^{c_{n}}
$$

Since $y_{1}(t), y_{2}(t), \ldots, y_{n}(t)$ are multiplicatively linear independent solutions of equation (19), these functions are $n$-times multiplicative differentiable. Hence, we can write

$$
\begin{gathered}
y^{*}=\left(y_{1}{ }^{*}\right)^{c_{1}}\left(y_{2}{ }^{*}\right)^{c_{2}}, \ldots,\left(y_{n}{ }^{*}\right)^{c_{n}} \\
\vdots \\
y^{*(n)}=\left(y_{1}{ }^{*(n)}\right)^{c_{1}}\left(y_{2}{ }^{*(n)}\right)^{c_{2}}, \ldots,\left(y_{n}{ }^{*(n)}\right)^{c_{n}}
\end{gathered}
$$

Substituting $y, y^{*}, \ldots, y^{*(n)}$ in equation (19), we get the next equality

$$
\begin{aligned}
L^{*}(\tilde{D}) y= & {\left[\left(y_{1}{ }^{*(n)}\right)^{c_{1}} \ldots\left(y_{n-1}{ }^{*(n)}\right)^{c_{n-1}}\left(y_{n}{ }^{*(n)}\right)^{c_{n}}\right]^{a_{n}}\left[\left(y_{1}{ }^{*(n-1)}\right)^{c_{1}} \ldots\left(y_{n-1}{ }^{*(n-1)}\right)^{c_{n-1}}\left(y_{n}{ }^{*(n-1)}\right)^{c_{n}}\right]^{a_{n-1}} } \\
& \ldots\left[\left(y_{1}{ }^{*}\right)^{c_{1}} \ldots\left(y_{n}\right)^{c_{n-1}}\left(y_{n}{ }^{*}\right)^{c_{n}}\right]^{a_{1}}\left[\left(y_{1}\right)^{c_{1}} \ldots\left(y_{n-1}\right)^{c_{n-1}}\left(y_{n}\right)^{c_{n}}\right]_{0}^{a_{0}} \\
= & {\left[\left(y_{1}{ }^{*(n)}\right)^{a_{n}} \ldots\left(y_{1}{ }^{{ }^{a_{1}}}\left(y_{1}\right)^{a_{0}}\right]^{c_{1}}\left[\left(y_{2}{ }^{*(n)}\right)^{a_{n}} \ldots\left(y_{2}{ }^{*}\right)^{a_{1}}\left(y_{2}\right)^{a_{0}}\right]^{c_{2}} \ldots\left[\left(y_{n}{ }^{*(n)}\right)^{a_{n}} \ldots\left(y_{n}{ }^{*}\right)^{a_{1}}\left(y_{n}\right)^{a_{0}}\right]^{c_{n}}\right.} \\
= & 1
\end{aligned}
$$

Theorem 8. Let $L^{*}(\tilde{D})$ be an operator with constant coefficients given by $(23)$ and $L(r)=\sum_{i=0}^{n} a_{i} r^{i}$. Then,

$$
L^{*}(\tilde{D})\left(e^{e^{r t}}\right)=\left(e^{e^{r t}}\right)^{L(r)}
$$

where $r$ is real or complex constant. 
Proof. For $y=e^{e^{r t}}$, We get

$$
\begin{gathered}
y^{*}=\tilde{D} y=e^{r e^{r t}} \\
y^{* *}=\tilde{D}^{2} y=e^{r^{2} e^{r t}} \\
\vdots \\
y^{*(n)}=\tilde{D}^{n} y=e^{r^{n} e^{r t}} .
\end{gathered}
$$

Substituting $y=e^{e^{r t}}$ and its multiplicative derivative in equation (22), then

$$
L^{*}(\tilde{D})\left(e^{e^{r t}}\right)=\left(e^{e^{r t}}\right)^{L(r)}
$$

is obtained. Hence, the proof is completed.

We seek the solution of (19) in the form $y=e^{e^{r t}}$, where $r$ is a real or complex constant. From equation (19) and Theorem 8 , It follows that

$$
L^{*}(\tilde{D})\left(e^{e^{r t}}\right)=\left(e^{e^{r t}}\right)^{L(r)}=(e)^{L(r) e^{r t}}=1=e^{0}
$$

For all $r$, we have $e^{r t} \neq 0$. Hence,

$$
L(r)=a_{n} r^{n}+a_{n-1} r^{n-1}+\ldots+a_{1} r+a_{0}=0
$$

is obtained. The equation (30) is called as characteristic equation of equation (19).

Theorem 9. If equation (30) have $n$ distinct roots $r_{1}, r_{2}, \ldots, r_{n}$, then the following functions

$$
y_{1}(t)=e^{e^{r_{1} t}}, y_{2}(t)=e^{e^{r_{2} t}}, \ldots, y_{n}(t)=e^{e^{r_{n} t}}
$$

are multiplicatively linear independent solutions of the equation (19). Since functions in (30) are multiplicatively linear independent solutions, the function

$$
y=\left(e^{e^{r_{1} t}}\right)^{c_{1}}\left(e^{e^{r_{2} t}}\right)^{c_{2}} \ldots\left(e^{e^{r_{n} t}}\right)^{c_{n}}
$$

or

$$
y=e^{c_{1} e^{r_{1} t}} e^{c_{2} e^{r_{2} t}} \ldots e^{c_{n} e^{r_{n} t}}
$$

Here, $e^{c_{1}}=C_{1}, e^{c_{2}}=C_{2}, \ldots, e^{c_{n}}=C_{n}$ and

$$
y=\left(C_{1}\right)^{e^{r_{1} t}}\left(C_{2}\right)^{e^{r_{2} t}} \ldots\left(C_{n}\right)^{e^{r_{n} t}}
$$

is the general solution of equation (19).

Proof. As equation $L(r)=0$ has $n$ distinct real roots $r_{i}, i=1,2, \ldots, n$, this equation can be written as

$$
L(r)=a_{n}\left(r-r_{1}\right)\left(r-r_{2}\right) \ldots\left(r-r_{n}\right)=0 .
$$

Hence equation (19) has $\mathrm{n}$ distinct solutions in the form $y=e^{e^{r t}}$ such as $y_{1}=e^{e^{r_{1} t}}, y_{2}=e^{e^{r_{2} t}}, \ldots, y_{n}=e^{e^{r_{n} t}}$. The multiplicatively Wronskian determinant of these solutions is

$$
W_{m}\left(y_{1}, y_{2}, \ldots, y_{n}\right)=\left|\begin{array}{cccc}
\ln y_{1} & \ln y_{2} & \ldots & \ln y_{n} \\
\ln y_{1}^{*} & \ln y_{2}^{*} & \ldots & \ln y_{n}^{*} \\
\vdots & \vdots & \ddots & \vdots \\
\ln y_{1}^{*(n-1)} & \ln y_{2}^{*(n-1)} & \ldots & \ln y_{n}^{*(n-1)}
\end{array}\right|=\left|\begin{array}{cccc}
e^{r_{1} t} & e^{r_{2} t} & \ldots & e^{r_{n} t} \\
r_{1} e^{r_{1} t} & r_{2} e^{r_{2} t} & \ldots & r_{n} e^{r_{n} t} \\
\vdots & \vdots & \ddots & \vdots \\
r_{1}^{(n-1)} e^{r_{1} t} & r_{2}{ }^{(n-1)} e^{r_{2} t} \ldots & r_{n}^{(n-1)} e^{r_{n} t}
\end{array}\right| \neq 0
$$


So, $y_{i}=e^{e^{r_{i} t}}, i=1,2, \ldots, n$ are multiplicatively linear independent solutions. Thus, fundamental set of solutions is

$$
T=\left\{e^{e^{r_{1} t}}, e^{e^{r_{2} t}}, \ldots, e^{e^{r_{n} t}}\right\}
$$

and the general solution is in the form $y_{h}=e^{c_{1} e^{r_{1} t}+c_{2} e^{r_{2} t}+\ldots+c_{n} e^{r_{n} t}}$.

Example 3. Consider the equation

$$
y^{* *}\left(y^{*}\right)^{3} y^{2}=1
$$

This equation corresponds to the equation

$$
\frac{d}{d t}\left(\frac{1}{y} \frac{d y}{d t}\right)+3\left(\frac{1}{y} \frac{d y}{d t}\right)=-2 \ln y
$$

or

$$
y^{\prime \prime} y-\left(y^{\prime}\right)^{2}+3 y y^{\prime}=-2 y^{2} \ln y
$$

The characteristic equation of the given equation is

$$
r^{2}+3 r+2=0
$$

The roots are $r_{1}=-2$ and $r_{2}=-1$. Hence, the general solution of equation (35), so equation (37) is

$$
y_{h}=\left(C_{1}\right)^{e^{-2 t}}\left(C_{2}\right)^{e^{-t}}
$$

Where $C_{1}=e^{c_{1}}$ ve $C_{2}=e^{c_{2}}$. Now, we consider characteristic equation (30) whose roots are not all distinct.

Theorem 10. If $m$ is a real root that appears $k$ times, that is, $L(r)=(r-m)^{k} p(r)$, where $p(m) \neq 0$, then for root- $m$,

$$
y_{h 1}=e^{\left(c_{1}+c_{2} t+c_{3} t^{2}+\ldots+c_{k} t^{k-1}\right)} e^{m t}
$$

is a solution of (19). For the remaining $n-k$ distinct roots,

$$
y_{h 2}=e^{c_{k+1} e^{m_{k+1} t}+c_{k+2} e^{m_{k+2} t}+\ldots+c_{n} e^{m_{n} t}} .
$$

is a solution of (19). Hence, the general solution of the equation (19) is

$$
y_{h}=y_{h 1} y_{h 2}=e^{\left(c_{1}+c_{2} t+c_{3} t^{2}+\ldots+c_{k} t^{k-1}\right) m t} e^{c_{k+1} e^{m_{k+1} t}+c_{k+2} e^{m_{k+2} t}+\ldots+c_{n} e^{m_{n} t}} .
$$

Proof. Suppose characteristic equation has $k$-times repeated real roots and has also distinct real roots. Then we can write

$$
L^{*}(\tilde{D}) y=e^{L(D)(\ln y)}=e^{\left[a_{n}(D-\alpha)^{k}\left(D-r_{k+1}\right) \ldots\left(D-r_{n}\right)\right](\ln y)}=e^{\left[F(D)(D-\alpha)^{k}\right](\ln y)}=1
$$

where

$$
F(D)=\left(D-r_{k+1}\right) \ldots\left(D-r_{n}\right)
$$

The solutions $y_{i}=e^{e^{r_{i} t}}=e^{e^{\alpha t}}, \quad i=1,2, \ldots, k$ with respect to $k$-times repeated roots $r_{i}=\alpha$ are not multiplicatively linear independent. But for $i=1,2, \ldots, k y_{i}=e^{t^{i-1} e^{\alpha t}}$ functions satisfy equation $e^{L(D)(\ln y)}=1$, in other words

$$
e^{L(D)\left(t^{i-1} e^{\alpha t}\right)}=e^{F(D)(D-\alpha)^{k} t^{i-1} e^{\alpha t}}=e^{F(D) e^{\alpha t} D^{k} t^{i-1}}=1 .
$$

Moreover, as multiplicatively Wronskian determinant of these functions is not equal to zero, they are multiplicatively linear independent. The first part of the proof is completed. For the second part the independence of solutions with respect 
to remaining distinct and real $(n-k)$ roots is given in the previous theorem. Finally,

$$
y_{1}=e^{e^{\alpha t}}, y_{2}=e^{t e^{\alpha t}}, \ldots, y_{k}=e^{t^{k-1} e^{\alpha t}}, y_{k+1}=e^{e^{r_{k+1} t}}, \ldots, y_{n}=e^{e^{r_{n} t}}
$$

The solutions with respect to $k$-times repeated roots are and $(n-k)$ real distinct roots are multiplicatively linear independent. The general solution is multiplicatively linear combination of these solutions with arbitrary constants.

Example 4. Consider the equation

$$
y^{* *}\left(y^{*}\right)^{-10} y^{25}=1
$$

The characteristic equation of the above equation is

$$
r^{2}-10 r+25=0
$$

The roots are $r_{1}=r_{2}=5$. Hence, the general solution is

$$
y_{h}=e^{\left(c_{1}+c_{2} t\right) e^{5 t}}
$$

Theorem 11. If $r_{1}=\alpha+i \beta, r_{2}=\overline{r_{1}}=\alpha-i \beta$ are conjugate complex roots of equation (19), which are each of multiplicity $k$, that is

$$
L(r)=\left(r-r_{1}\right)^{k}\left(r-\overline{r_{1}}\right)^{k} p(r)
$$

where $p\left(r_{1}\right) \neq 0, p\left(\overline{r_{1}}\right) \neq 0 . y_{1}=e^{e^{r_{1} t}}$ and $y_{2}=e^{e^{r_{2} t}}$ are multiplicatively linear independent solutions. Hence, general solution is

$$
y_{h}=e^{\left[C_{1} \cos \beta t+C_{2} \sin \beta t\right] e^{\alpha t}+\ldots+\left[C_{2 k-1} \cos \beta t+C_{2 k} \sin \beta t\right] t^{(k-1)} e^{\alpha t}+C_{2 k+1} e^{m_{2 k+1} t}+\ldots+C_{n} e^{m_{n} t}} .
$$

Proof. By Theorem 10, the solution is

$$
y_{1}=e^{\left(c_{1}+c_{2} t+\ldots+c_{k} t^{(k-1)}\right)} e^{(\alpha+i \beta) t}
$$

for $\mathrm{k}$-times repeated root $r_{1}=\alpha+i \beta$ and the solution is

$$
y_{2}=e^{\left(c_{k+1}+c_{k+2} t+\ldots+c_{2 k} t^{(k-1)}\right) e^{(\alpha-i \beta) t}}
$$

$\overline{r_{1}}=\alpha-i \beta$. For the remaining $n-k$ distinct roots we have solution

$$
y_{3}=e^{c_{2 k+1} e^{m_{2 k+1} t}+c_{2 k+2} e^{m_{2 k+2} t}+\ldots+c_{n} e^{m_{n} t}}
$$

Each of $y_{1}, y_{2}, y_{3}$ solutions are multiplicatively linear independent. The linear combination of these solutions is the general solution of the equation (19) which can be stated as

$$
\begin{gathered}
y_{h}=y_{1} y_{2} y_{3} \\
y_{h}=e^{\left(c_{1}+c_{2} t+\ldots+c_{k} t^{(k-1)}\right) e^{(\alpha+i \beta) t}} e^{\left(c_{k+1}+c_{k+2} t+\ldots+c_{2 k} t^{(k-1)}\right) e^{(\alpha-i \beta) t}} e^{c_{2 k+1} e^{m_{2 k+1} t}+c_{2 k+2} e^{m_{2 k+2} t}+\ldots+c_{n} e^{m_{n} t}}
\end{gathered}
$$

If we use following Euler equations,

$$
\begin{gathered}
e^{i \beta t}=\cos \beta t+i \sin \beta t \\
e^{-i \beta t}=\cos \beta t-i \sin \beta t
\end{gathered}
$$

then the general solution of the equation (19) can be written as

$$
y_{h}=e^{\left.\left[c_{1} \cos \beta t+c_{2} \sin \beta t\right] e^{a t}+\ldots+\left[c_{2 k-1} \cos \beta t+c_{2 k} \sin \beta t\right]\right]^{k-1} e^{a t}+c_{2 k+1} e^{m_{2 k+1} t}+\ldots+c_{n} e^{m_{n} t}} .
$$


which is multiplicatively linear independent. Here, if we use notations

$$
c_{1}+c_{k+1}=C_{1}, i\left(c_{1}-c_{k+1}\right)=C_{2}, \ldots, c_{k}+c_{2 k}=C_{2 k-1}, i\left(c_{k}-c_{2 k}\right)=c_{2 k},
$$

then the general solution of the equation (19) can be rewritten as

$$
y_{h}=e^{\left[C_{1} \cos \beta t+C_{2} \sin \beta t\right] e^{\alpha t}+\ldots+\left[C_{2 k-1} \cos \beta t+C_{2 k} \sin \beta t\right] t^{(k-1)} e^{\alpha t}+C_{2 k+1} e^{m_{2 k+1} t}+\ldots+C_{n} e^{m_{n} t} .}
$$

Example 5. Consider the equation

$$
y^{* *} y^{*} y=1
$$

The characteristic equation of the above equation is

$$
r^{2}+r+1=0
$$

The roots are

$$
\begin{aligned}
& r_{1}=\frac{-1}{2}+i \frac{\sqrt{3}}{2}, \\
& r_{2}=\frac{-1}{2}-i \frac{\sqrt{3}}{2}
\end{aligned}
$$

Hence, the general solution is

$$
y_{h}=e^{\frac{-t}{2}\left[c_{1} \cos \left(\frac{\sqrt{3}}{2} t\right)+c_{2} \sin \left(\frac{\sqrt{3}}{2} t\right)\right]}
$$

\section{Conclusion}

In this study, the relation between multiplicatively Wronskian determinant and multiplicatively linear independent is given for positive definite functions after defining the multiplicatively linear independent, multiplicatively linear dependent and multiplicatively Wronskian determinant. On the other hand, multiplicative homogeneous differential equations with constant exponentials are defined and the solutions are obtained. Solutions for these equations that have correspondence with a class of non-linear differential equations in the classical sense are stated.

\section{Competing interests}

The authors declare that they have no competing interests.

\section{Authors' contributions}

All authors have contributed to all parts of the article. All authors read and approved the final manuscript.

\section{References}

[1] M. Grossman, R. Katz, Non-Newtonian Calculus, Lee Press, Pigeon Cove, MA, 1972.

[2] D. Stanley. A multiplicative calculus, Primus, 1999, IX(4), 310-326.

[3] A. E. Bashirov, E. Misırlı and A. Özyapıcı, Multiplicative calculus and its applications, J. Math. Anal. Appl., 2008, 337, 36-48

[4] A. E. Bashirov, E. Mısırlı, Y. Tandoğdu and A. Özyapıcı, On modeling with multiplicative differential equations, Applied Mathematics-A Journal of Chinese Universities, 2011, 26(4), 425-438 
[5] L. Florack and H. Assen, Multiplicative Calculus in Biomedical Image Analysis, J Math Imaging Vis. 2012, 42, 64-75

[6] D. A. Filip and C. Piatecki. A non-Newtonian examination of the theory of exogenous economic growth. Mathematica Aeterna, 2014.

[7] M. Mora, F. Cordova-Lepe and R. Del-Valle, A non-Newtonian gradient for contour detection in images with multiplicative noise, Pattern Recognition Letters, 2012, 33, 1245-1256

[8] D. Filip and C. Piatecki, An overview on the non-newtonian calculus and its potential applications to economics, Applied Mathematics and Computation, 2007, 187(1), 68-78.

[9] Aniszewska, D., Multiplicative Runge-Kutta method, Nonlinear Dynamics, 2007, 50, 265-272.

[10] A. Özyapıcı, Çarpımsal Analiz ve Uygulamaları., Ege University PhD thesis,2009.

[11] N. Yalcin, E. Celik, and A. Gokdogan, Multiplicative Laplace transform and its applications, Optik - International Journal for Light and Electron Optics, 2016, 127(20): 9984-95.

[12] N. Yalçın, and E. Çelik,The Solution of Multiplicative Non-Homogeneous Linear Differential Equations,.Journal of Applied Mathematics and Computation, 2018, 2(1): 27-36 